Iranian Journal of Pathology | ISSN: 2345-3656

\title{
Intestinal Infarction in COVID-19 Pandemic: A Case Series
}

\author{
Maryam Sarkardeh ${ }^{1,2}$, Amin Dalili' ${ }^{1,2}$, Naser Tayyebi Meibodi ${ }^{3}$, Mostafa Izanlu ${ }^{3 *}$, \\ Seyed Javad Davari-Sani ${ }^{4}$, Saeed Moghaddamzade ${ }^{2}$, Mehdi Jamalinik ${ }^{5}$, Seyed Javad Hosseini ${ }^{6}$, \\ Javad Koushki ${ }^{2}$, Ali Abedi ${ }^{6}$
}

1. Surgical Oncology Research Center, Faculty of Medicine, Mashhad University of Medical Sciences, Mashhad, Iran

2. Department of Surgery, Imam Reza Hospital, Mashhad University of Medical Sciences, Mashhad, Iran

3. Department of Pathology, Imam Reza Hospital, Mashhad University of Medical Sciences, Mashhad, Iran

4. Student Research Committee, Sabzevar University of Medical Sciences, Sabzevar, Iran

5. Department of Nursing, Islamic Azad University, Tabas Branch, Tabas, Iran

6. Department of Nursing, Esfarayen Faculty of Medical Sciences, Esfarayen, Iran

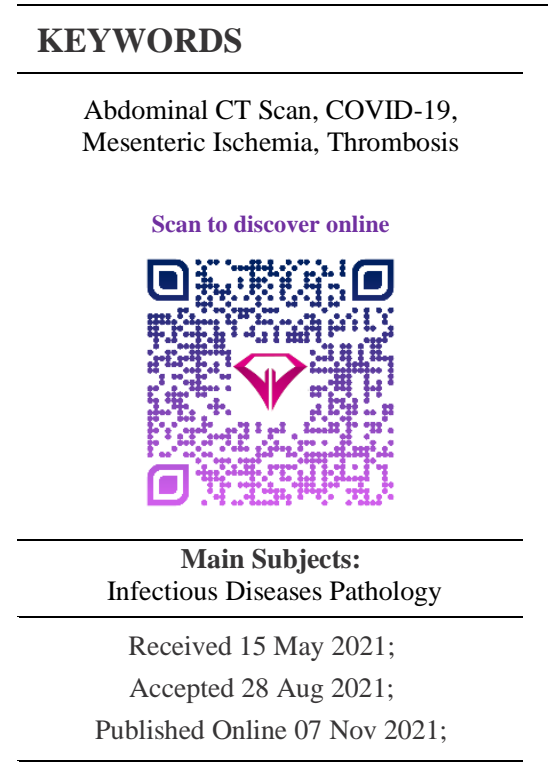

\section{ABSTRACT}

Novel coronavirus disease 2019 (COVID-19) as a potential health risk factor continues to spread worldwide. Although common symptoms include headache and respiratory symptoms, some studies have suggested that COVID-19 may cause coagulation disorders and thrombolytic events, disrupt blood flow to the visceral organs, and cause some complications such as mesenteric ischemia. The authors reported four cases of acute mesenteric ischemia associated with COVID-19 confirmed in patients hospitalized in Imam Reza Hospital (a COVID-19 referral center in Mashhad University of Medical Sciences, Mashhad, Iran). The authors described the pathological findings that may be associated with this infection. The authors collected clinical data, imaging, microscopic, and operative findings of four patients with severe COVID-19 infection and evidence of intestinal necrosis. These four cases of severe COVID-19 pneumonia simultaneously showed intestinal necrosis during the infection process, indicating a relationship between coronavirus and mesenteric vascular events. Physicians should be aware of thrombosis symptoms in the digestive system in patients with severe COVID-19.

\section{$\underline{10.30699 / I J P .2021 .525280 .2600 ~}$}

\section{Corresponding Information:}

Mostafa Izanlu, Department of Pathology, Imam Reza Hospital, Mashhad University of Medical Sciences, Mashhad, Iran. Email: mostafa.izanlu@gmail.com

Copyright $(\odot$ 2022. This is an open-access article distributed under the terms of the Creative Commons Attribution- 4.0 International License which permits Share, copy and redistribution of the material in any medium or format or adapt, remix, transform, and build upon the material for any purpose, even commercially.

\section{Introduction}

Novel coronavirus disease 2019 (COVID-19) is now a worldwide pandemic caused by severe acute respiratory syndrome coronavirus 2 (SARS-CoV-2) (1). New evidence suggests that venous and arterial thromboembolic disease due to hypoxia and hyperinflammatory response to COVID-19 may lead to the extensive amount of inflammatory cytokines and activation of different coagulation pathways (2). Also, in patients with severe COVID-19, mild to moderate lower platelet count (thrombocytopenia), elevated D-dimer, increased fibrin destruction products, and prolonged prothrombin time have been reported (3). Numerous studies have suggested that there is a link between gastrointestinal symptoms that are seen in patients with COVID-19 and coagulation disorders such as excessive coagulation and intestinal necrosis (4). Intestinal necrosis is a late symptom of various disease processes, which eventually causes cell death as a result of reduced blood flow to the bowel. These severe and deadly conditions are often caused by acute vascular occlusion and, less commonly, bowel obstruction infection or inflammation (5). As a result, based on the findings, hypercoagulable state, blood stasis, and endothelial injury increase the risk of thrombosis in patients with COVID-19 (6).

\section{Case Report and Methods}

In this case series, we presented four cases infected with COVID-19 confirmed by reverse transcriptasepolymerase chain reaction (RT-PCR); they were referred to Imam Reza Hospital Complex, Mashhad, Iran, with abdominal discomforts; the laboratory tests, imaging, pathology, and operating findings suggested intestinal necrosis.

\section{Case 1}

A 58-year-old man with COVID-19 confirmed by RT-PCR and chest computed tomography (CT) scan 
(Figure 1) was referred to the emergency department with a history of mitral valve replacement, hypertension, and complaint of generalized abdominal pain and dyspnea. Vital signs on admission time were as follows: blood pressure (BP) $140 / 70 \mathrm{~mm} \mathrm{Hg}$, pulse rate (PR) $120 / \mathrm{min}$, temperature $37.3^{\circ} \mathrm{C}$ (oral), and oxygen saturation $\left(\mathrm{SpO}_{2}\right) 92 \%$ in room air. He had a relative decrease in white blood cell (WBC) count. On abdominal examination, he had tenderness in the right lower quadrant (RLQ) without any guarding and rebound tenderness. He used warfarin due to cardiac problems; the international normalized ratio (INR) was 8 upon arrival, and no cardiac thrombosis was seen on his echocardiogram. After an abdominal CT scan due to abdominal pain, mesenteric ischemia was diagnosed. After correction of INR with transfusion of fresh frozen plasma (FFP), surgery was planned for him. During the surgery, $140 \mathrm{~cm}$ of the small intestine in the ileum was ischemic (Figures $\underline{2}$ and $\underline{3}$ ). After the operation, he suffered a drop in $\mathrm{SpO}_{2}$ and $\mathrm{BP}$ that led to his death.

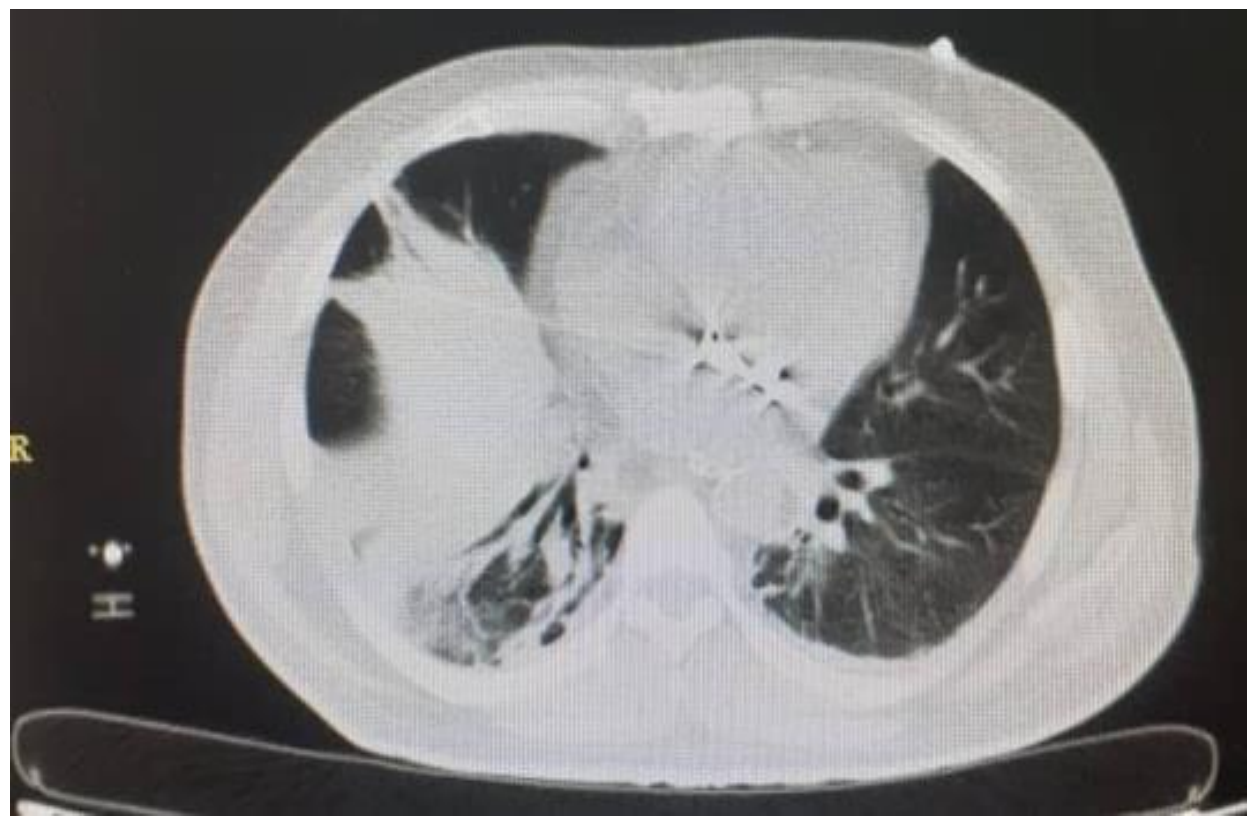

Fig. 1. Lung involvement with COVID-19

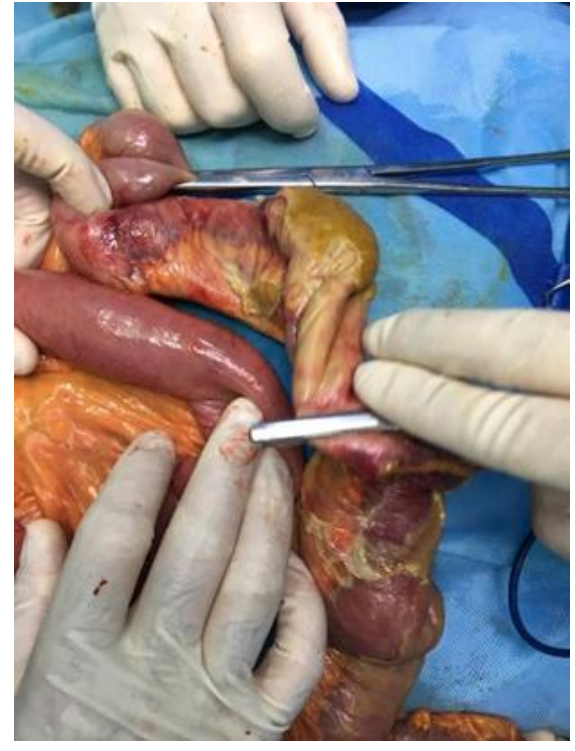

Fig. 2. Intestinal ischemia and necrosis that it was resected

\section{Case 2}

A 51-year-old man with confusion and a history of diabetes and end-stage renal disease (ESRD, who was on dialysis treatment for years) was referred to the emergency department. On arrival to the emergency

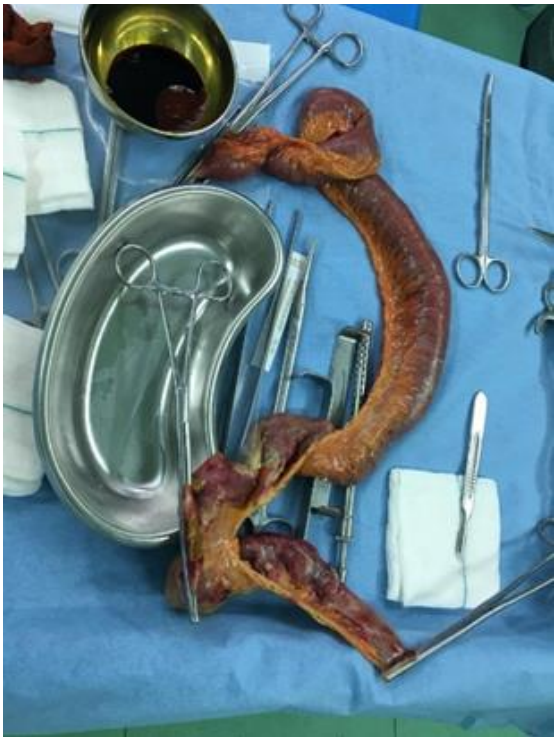

Fig. 3. Intestinal ischemia and necrosis that it was resected

department, he had a fever, respiration distress, low $\mathrm{SpO}_{2}$ level (92\% with additional oxygen), and abdominal pain. High-resolution computed tomography (HRCT) was performed due to the patient's 
dyspnea, which was in favor of COVID-19 (Figure 4), and two days after the PCR test, it was reported positive. Blood tests also showed high levels of amylase, INR, Partial Thromboplastin Time (PTT), lactate dehydrogenase (LDH), and thrombocytopenia $(75,000 / \mu \mathrm{L})$. In the primary complete blood cell count (CBC) test, the hemoglobin level was $10.9 \mathrm{mg} / \mathrm{dL}$. In the lower cuts of HRCT, free air (pneumoperitoneum) was seen. The patient underwent an emergency laparotomy; during the operation, necrosis and perforation in three parts of the ileum with a length of $40 \mathrm{~cm}$ at a distance of $70 \mathrm{~cm}$ from the ileocecal valve were seen; after the operation, the patient's condition became unstable that led to his death.

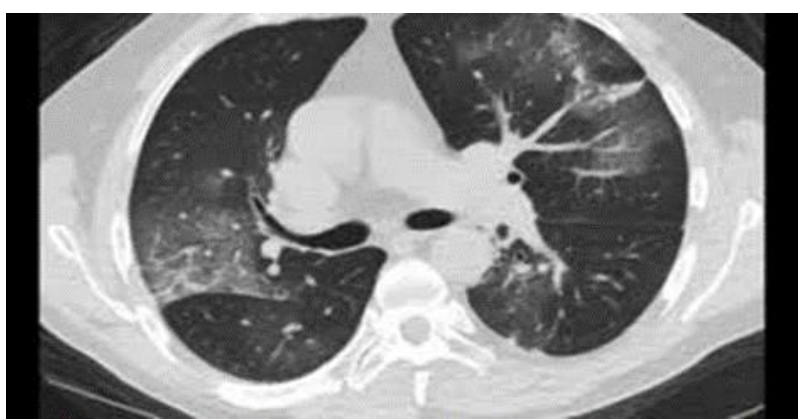

Fig. 4. lung involvement with COVID-19

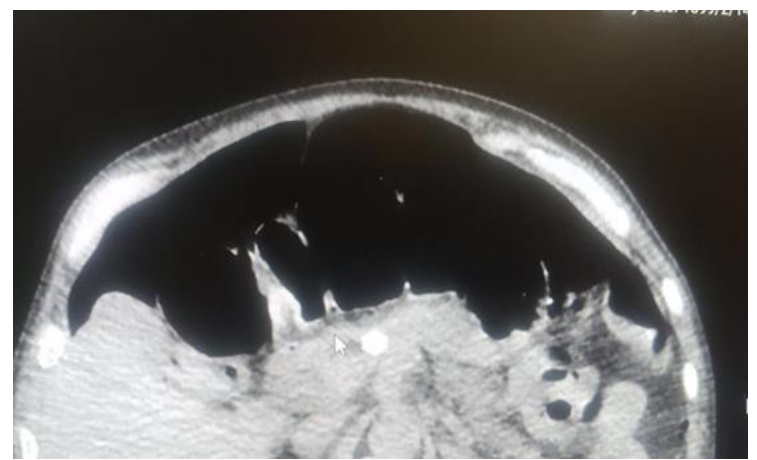

Fig. 5. Pneumoperitoneum in CT scan

Case 4

An 88-year-old man with a diagnosis of COVID-19 confirmed by PCR was referred to the emergency department due to respiratory distress from one week ago and melena (several times) from three days ago, abdominal pain, bloating, and inability to expel gas and excrete feces. During the abdominal examination, he had generalized tenderness, rebound tenderness, and abdominal distention. He had anemia and low hemoglobin and hematocrit, and the levels of urea and creatinine were high. Considering abdominal examination and final assessment (Figure 7), a diagnosis of peritonitis was made. Accordingly, the patient was prepared for emergency surgery; during the operation, about $130 \mathrm{~cm}$ of the ileum was resected due to necrosis. Unfortunately, two days after surgery, the patient died due to respiratory arrest.

\section{Case 3}

A 60-year-old man was admitted to the intensive care unit (ICU) due to COVID-19 with no past medical history, and infection was confirmed by the COVID-19 PCR test. Four days after admission, the patient complained of abdominal pain and inability to expel gas and excrete feces. The patient's consciousness decreased the day before the surgical consultation. The surgeon reported tenderness and rebound tenderness during the physical examination. Free air was confirmed by an abdominal CT scan (Figure 5). Blood tests also determined a mild decrease in platelet $(130,000 / \mu \mathrm{L})$ and an increase in Prothrombin time (PT), Partial thromboplastin time (PTT), international normalized ratio (INR), lactate dehydrogenase (LDH)

bill direct, Aspartate aminotransferase (AST), and Alanine aminotransferase (ALT). Due to signs of peritonitis and free air in imaging, the patient underwent emergency surgery. In this regard, a midline laparotomy was performed, and macroscopic observations revealed that a $60-\mathrm{cm}$-long intestine near the ileocecal valve and entire colon was necrosis (Figure 6). Unfortunately, two days after surgery, the patient died due to respiratory arrest and hypotension.

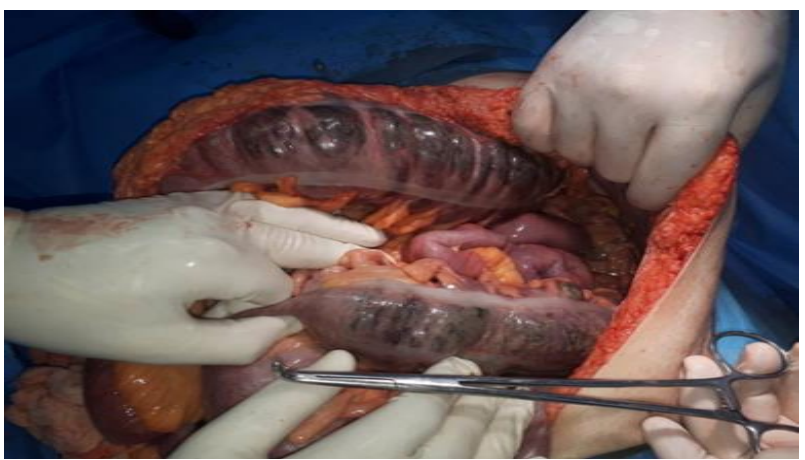

Fig. 6. intestinal ischemia and necrosis

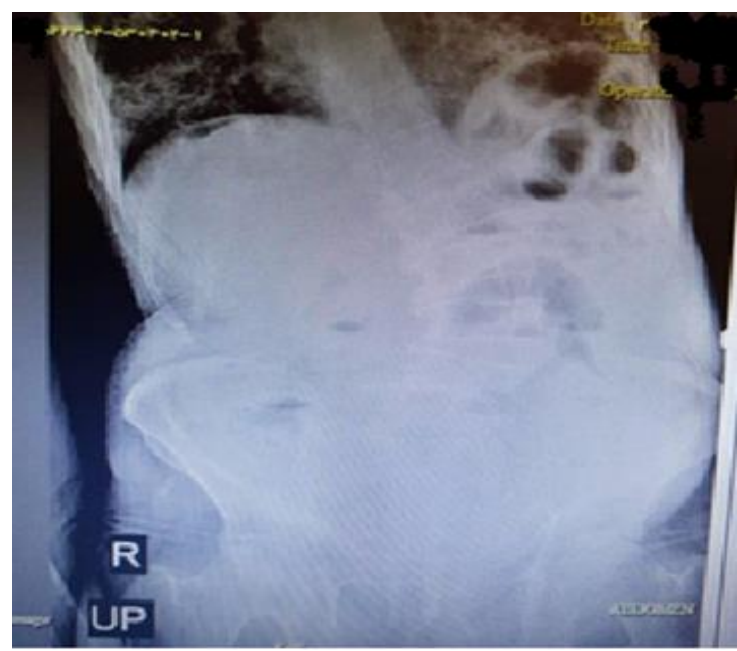

Fig. 7. Abdominal X-ray, upright and supine 


\section{Histopathological Findings}

The macroscopic and microscopic findings were same in all cases. All samples in the gross description presented with segmental thinning in areas of fullthickness infarction, and the outer surface was brown and had a congestive appearance. A luminal surface with multiple foci of mucosal ulceration was reported (Figure 8).
In the histopathologic evaluation, microscopic findings showed identical results, perforation in three cases, multiple ulcerations, coagulative and liquefactive transmural necrosis, intraluminal thrombus with vascular wall, and perivascular prominent neutrophilic infiltration (Figures 9-11).

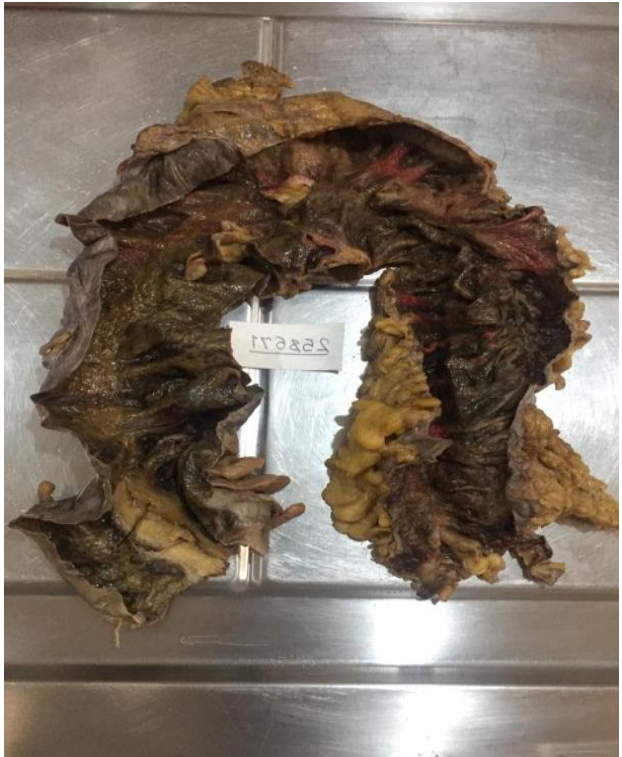

Fig. 8. The outer surface was brown and congestive appearance, segmental thinning in areas of fullthickness infarction, luminal surface with multiple foci of mucosal ulceration

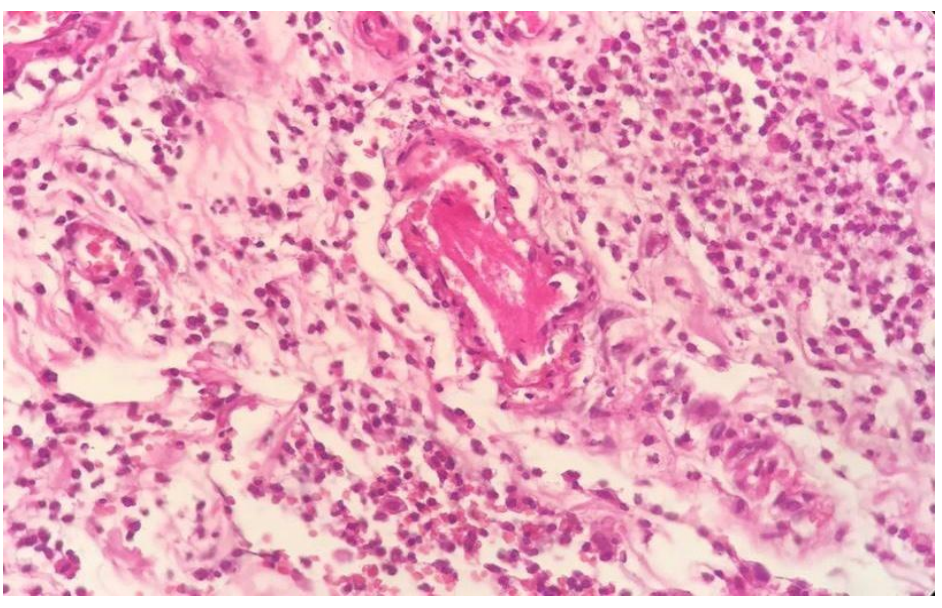

Fig. 11. Intraluminal thrombus and perivascular prominent neutrophilic infiltration

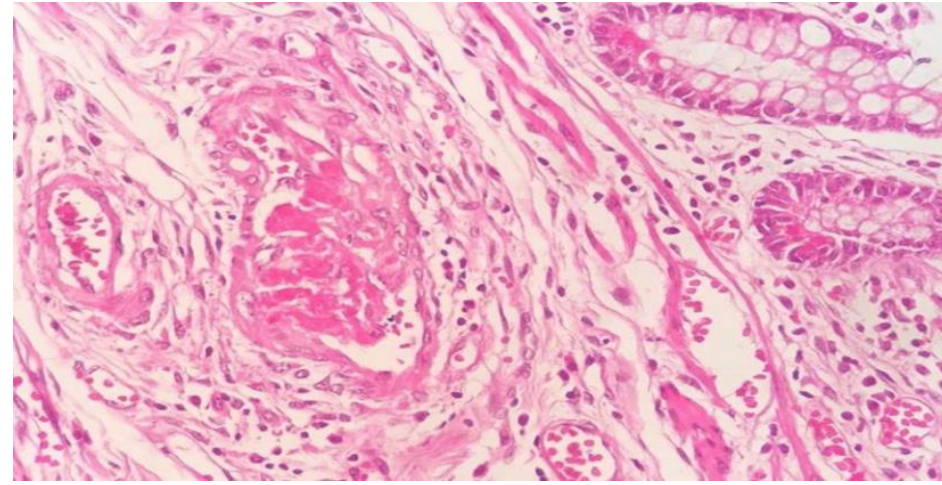

Fig. 9. Intraluminal thrombus with vascular wall and perivascular neutrophilic infiltration

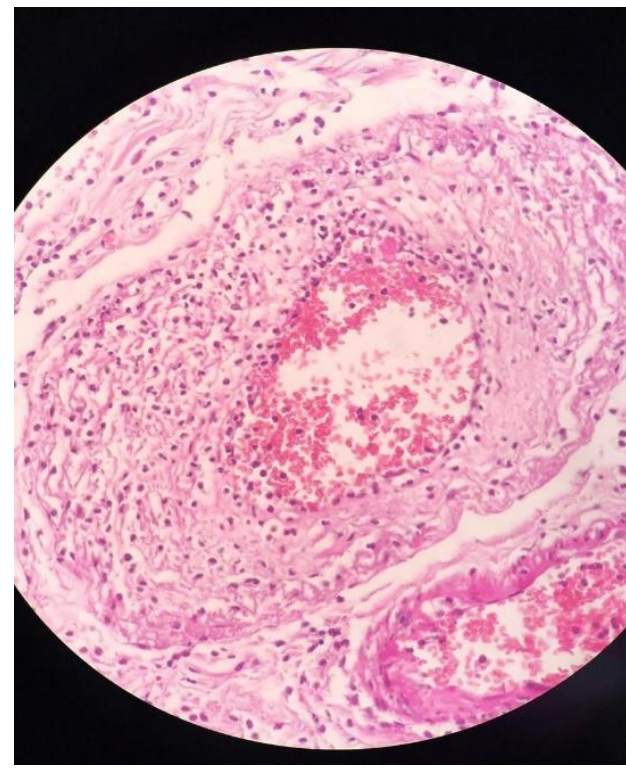

Fig. 10. vascular wall and perivascular neutrophilic infiltration

\section{Discussion}

Recently published COVID-19 studies have shown that coagulation abnormalities occur in patients with COVID-19, including hypercoagulation status, thromboembolic disease, and ischemia $(7,8)$.

The pathophysiology of hypercoagulability in COVID-19 is vascular endothelial dysfunction, causing microcirculatory changes in SARS-CoV-2 (7, $8)$. The virus adheres to a receptor by the angiotensinconverting enzyme 2 (ACE2) receptor on endothelial cells (9). Then, viral replication causes inflammatory cell infiltration, endothelial cell apoptosis, and microvascular prothrombotic effects (9). Polyphosphates formed from viral antigens activate platelets, 
mast cells, and factor XII, and they activate other coagulation pathways as well (10).

Ignat et al. reported a mesenteric and portal vein thrombosis in patients with SARS-CoV-2 (11). Rossana et al. indicated abnormalities in medium and small size pulmonary vessels in COVID-19 patients due to perivascular inflammation; endothelial cells appeared large, massive liquefactive degeneration of endothelial cells, multiple intraluminal thrombi (12). Louis Maximilian Buja et al. collected pathological findings from 23 autopsies; they reported several cardiopulmonary events due to microthrombosis in small lung vessels and intravascular trapping of neutrophils and endothelial damages in heart and lung tissues (13).

Guidelines suggest that patients with COVID-19 infection should receive prophylactic doses with lowmolecular-weight heparin (LMWH) unless there is a contraindication to anticoagulant therapy (13). The question now is whether thromboprophylaxis should be used in outpatients. Several studies have reported that for patients with thrombotic risk factors (such as venous thromboembolism [VTE], immobilization, and recent surgery), anticoagulant therapy is useful (14).

Consistent with several recent studies, these four COVID-19 cases showed intestinal necrosis during the infection process, which may indicate a relationship between coronavirus and mesenteric vascular events. However, further studies with more cases are needed to confirm this relationship.

\section{Conclusion}

These cases showed that prothrombotic consequences of COVID-19 are arterial and venous abdominal thrombosis, especially in severe cases. Therefore, in SARS-CoV-2, more attention should be paid to arterial and venous abdominal thrombosis, even in the absence of obvious signs of intravascular coagulation. In this paper, the cases had an underlying risk factor in addition to COVID-19, and the role of other risk factors cannot be ignored; accordingly, further studies with a larger sample size are needed to investigate the relationship of other risk factors with COVID-19.

\section{Acknowledgments}

The authors would like to thank Mashhad University of Medical Sciences for financial support

\section{Conflict of Interest}

The authors declared no conflicts of interest.

\section{References}

1. Coronavirus disease (COVID-19) Weekly Epidemiological Update and Weekly Operational Update [Available from:

https://www.who.int/emergencies/diseases/novelcoronavirus-2019/situation-reports

2. Merad M, Martin JC. Pathological inflammation in patients with COVID-19: a key role for monocytes and macrophages. Nature reviews immunology. 2020;20(6):355-62. [DOI:10.1038/s41577-0200331-4] [PMID] [PMCID]

3. Lippi G, Plebani M, Henry BM. Thrombocytopenia is associated with severe coronavirus disease 2019 (COVID-19) infections: A meta-analysis. Clin Chim Acta. 2020;506:145-8. [PMID] [PMCID] [DOI:10.1016/j.cca.2020.03.022]

4. Fabre O, Rebet O, Carjaliu I, Radutoiu M, Gautier L, Hysi I. Severe Acute Proximal Pulmonary Embolism and COVID-19: A Word of Caution. Ann Thorac Surg. 2020;110(5):e409-e11. [DOI:10.1016/j.athoracsur.2020.04.005] [PMID] [PMCID]

5. Clair DG, Beach JM. Mesenteric Ischemia. N Engl J Med. 2016;374(10):959-68. [DOI:10.1056/NEJMra1503884] [PMID]
6. Zhou B, She J, Wang Y, Ma X. Venous thrombosis and arteriosclerosis obliterans of lower extremities in a very severe patient with 2019 novel coronavirus disease: a case report. J Thromb Thrombolysis. 2020;50(1):229-32. [PMID] [PMCID] [DOI:10.1007/s11239-020-02084-w]

7. Cui S, Chen S, Li X, Liu S, Wang F. Prevalence of venous thromboembolism in patients with severe novel coronavirus pneumonia. J Thromb Haemost. 2020;18(6):1421-4. [DOI:10.1111/jth.14830] [PMID] [PMCID]

8. Iba T, Levy JH. Derangement of the endothelial glycocalyx in sepsis. J Thromb Haemost. 2019;17 (2):283-94. [DOI:10.1111/jth.14371] [PMID]

9. Varga Z, Flammer AJ, Steiger P, Haberecker M, Andermatt R, Zinkernagel AS, et al. Endothelial cell infection and endotheliitis in COVID-19. Lancet. 2020;395(10234):1417-8. [DOI:10.1016/S0140-6736(20)30937-5]

10. Smith SA, Mutch NJ, Baskar D, Rohloff P, Docampo R, Morrissey JH. Polyphosphate modulates blood coagulation and fibrinolysis. Proc Natl Acad Sci U S A. 2006;103(4):903-8. [PMID] [DOI:10.1073/pnas.0507195103] [PMCID] 
11. Ignat M, Philouze G, Aussenac-Belle L, Faucher V, Collange O, Mutter D, et al. Small bowel ischemia and SARS-CoV-2 infection: an underdiagnosed distinct clinical entity. Surgery. 2020;168(1):14-6. [DOI:10.1016/j.surg.2020.04.035] [PMID] [PMCID]

12. Bussani R, Schneider E, Zentilin L, Collesi C, Ali $\mathrm{H}$, Braga L, et al. Persistence of viral RNA, pneumocyte syncytia and thrombosis are hallmarks of advanced COVID-19 pathology. EBioMedicine. 2020;61:103104. [PMID] [PMCID] [DOI:10.1016/j.ebiom.2020.103104]
13. Buja LM, Wolf DA, Zhao B, Akkanti B, McDonald $\mathrm{M}$, Lelenwa L, et al. The emerging spectrum of cardiopulmonary pathology of the coronavirus disease 2019 (COVID-19): Report of 3 autopsies from Houston, Texas, and review of autopsy findings from other United States cities. Cardiovasc Pathol. 2020;48:107233. [PMID] [PMCID] [DOI:10.1016/j.carpath.2020.107233]

14. Emert R, Shah P, Zampella JG. COVID-19 and hypercoagulability in the outpatient setting. Thromb Res. 2020;192:122-3. [PMID] [PMCID] [DOI:10.1016/j.thromres.2020.05.031] 